\title{
USING THE PRINCIPLES OF IMAGE ANALYSIS IN THE ASSESSMENT OF THE PROPORTION OF RETAINED AUSTENITE IN THE CASE HARDENED LAYERS
}

\begin{abstract}
Structures of carburized layers after the surface saturation process in gaseous, liquid or solid medium and after subsequent heat treatment (hardening and low-temperature tempering) consist mainly of high carbon plate martensite with a certain portion of retained austenite. The presence of retained austenite (RA) in carburized layers is mostly considered as undesirable because it decreases hardness of the hardened layer and furthermore, a spontaneous conversion to a ferritic-carbide mixture of a bainitic type, accompanied by a change of properties, dimensional instability and the local increase in internal stress with the possible formation of cracks, can occur. The proportion of retained austenite is, therefore, a significant characteristics of the quality of hardened layers. This work deals with the evaluation of the volume fraction of retained austenite in carburized layers using image analysis on metallographic images.
\end{abstract}

Keywords: retained austenite, carburized layers, image analysis

\section{Introduction}

The structure of carburized layers after the saturation process of the surface of parts in gaseous, liquid or solid medium and subsequent heat treatment (quenching and low-temperature tempering) is formed by high carbon plate martensite with a certain proportion of untransformed austenite. Austenite, which is preserved in the structure of the hardened steel, is referred to as retained austenite. Its presence after the quenching in a bath depends on the temperature range of martensitic transformation, on the possibility of austenite stabilization and on the austenite grain size. The amount of retained austenite after quenching in a bath of room temperature increases rapidly with the carbon content and alloying elements. The proportion of retained austenite also increases with decreasing austenite grain size and with a decreasing cooling rate, so the proportion is the greatest at the critical cooling rate $[1,2]$.

The presence of retained austenite in carburized layers is considered generally undesirable because it reduces the hardness of the hardened layer and can lead to spontaneous transformation to a ferritic-carbidic mixture of a bainitic type which is accompanied by a change of properties, dimensional instability and the local increase of internal stress with the possible formation of cracks. The proportion of retained austenite is, therefore, an important characteristics of the quality of carburized layers [1,2].

The basic methods of studying the structure of solid crystalline materials include light microscopy (metallographic evaluation), methods using a focused electron beam, e.g. electron backscattered diffraction (EBSD) and X-ray diffraction
[4-8]. This work deals with the evaluation of volume fraction of retained austenite in carburized layers using image analysis on metallographic images.

Taking metallographic images in digital format allowed the development of automated image analysis. Individual pixels are disclosed by a single numerical value so-called gray level. 8-bit display, which is equivalent to 256 gray levels, is usually used as the default. Absolutely white is associated with gray level 255 and absolute black gray level 0 . From the histogram of gray levels can be seen, what is the appearance frequency of different gray levels in the image. If the histogram of gray levels of images shows at least two maxima, the detection of areas in the image by so-called thresholding is possible. The image is then suitable for quantitative analysis. The quality of the scanned image and other image operations affect the distribution of gray levels in the histogram and therefore affect the thresholding value. The actual value of thresholding then influences the resulting values of the quantitative proportion of phases $[3,4]$.

The influence of various parameters of image adjustments on the final retained austenite content in carburized layers will be evaluated in the following experimental section.

\section{Experimental methods}

A carburized piece was used for the evaluation of retained austenite content: a gear wheel was made of steel $20 \mathrm{MnCr} 5$. The carburizing was carried out in a gaseous atmosphere at a temperature of $920^{\circ} \mathrm{C}$, followed by quenching from a tem-

\footnotetext{
* VŠB - TECHNICAL UNIVERSITY OF OSTRAVA, 17. LISTOPADU 15/2172, 70833 OSTRAVA - PORUBA, CZECH REPUBLIC

\# Corresponding author: petra.vanova@vsb.cz
} 
perature of $820^{\circ} \mathrm{C}$ to a salt bath and tempering at a temperature of $160^{\circ} \mathrm{C}$. The assessment of retained austenite content was carried out on a metallographic sample at a depth of $0.1 \mathrm{~mm}$; the sample was repeatedly etched in $4 \%$ Nital (Fig. 1). The quality of metallographic samples and etching rate plays a significant role in the evaluation of images using digital image analysis.

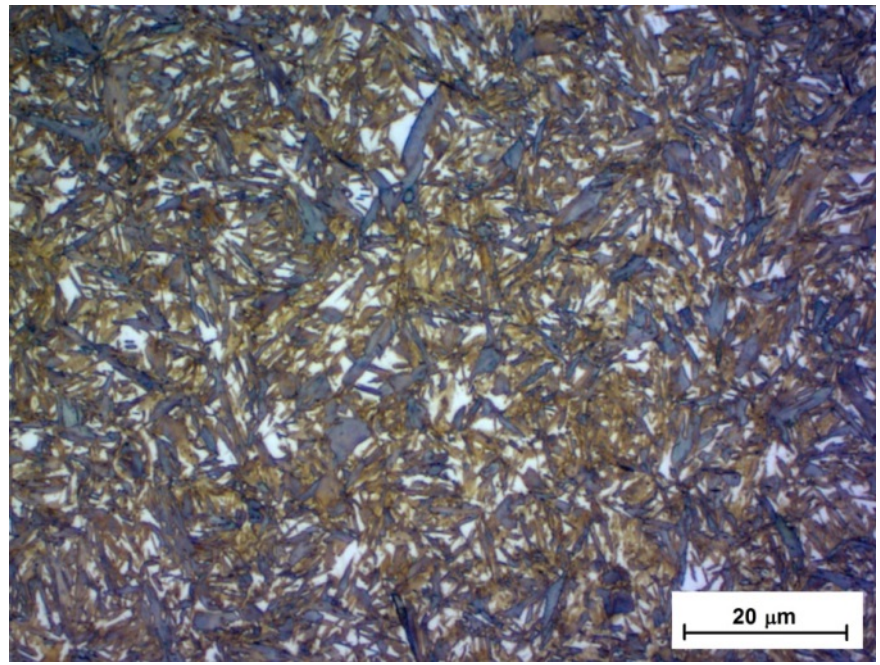

Fig. 1. The microstructure of the carburized layer in the depth of $0.1 \mathrm{~mm}$ under the surface (Nital etch)
The documentation of the carburized microstructure was taken by the metallographic microscope Zeiss Axio Observer A1m and Axio Vision 4.8 software at the magnification 1000x. The retained austenite content was evaluated from photographs with no other digital image editing and also from photographs which were edited using delinearizing filter in advance. The delinearization was carried out using the computer program Gray Level Analyzer, which includes this kind of digital filtration. The size of the matrix was set to $5 \times 5$ and the threshold for the Sobel operator to a value of 30 (Sobel filtering was used in order to identify martensite / retained austenite interface). The influence of delinearizing filter is apparent from Fig. 2 and Fig. 3. Gray levels in martensite / retained austenite interface change more steeply when using the delinearizing filter (Fig. 3) in comparison with the slower profile of gray levels in the original not delinearized image (Fig. 2). The image editing by delinearization then allowed better image thresholding and simultaneously the influence of the thresholding value decreased, as will be stated below (Fig. 4 and Fig. 5).

The computer program Image-Pro Analyzer 7.0 was used to determine the retained austenite content. In this program, images were transferred to the 8-bit range of 256 gray scales. The brightness and contrast of original photographs were not changed. In some cases, the gamma correction of the image from
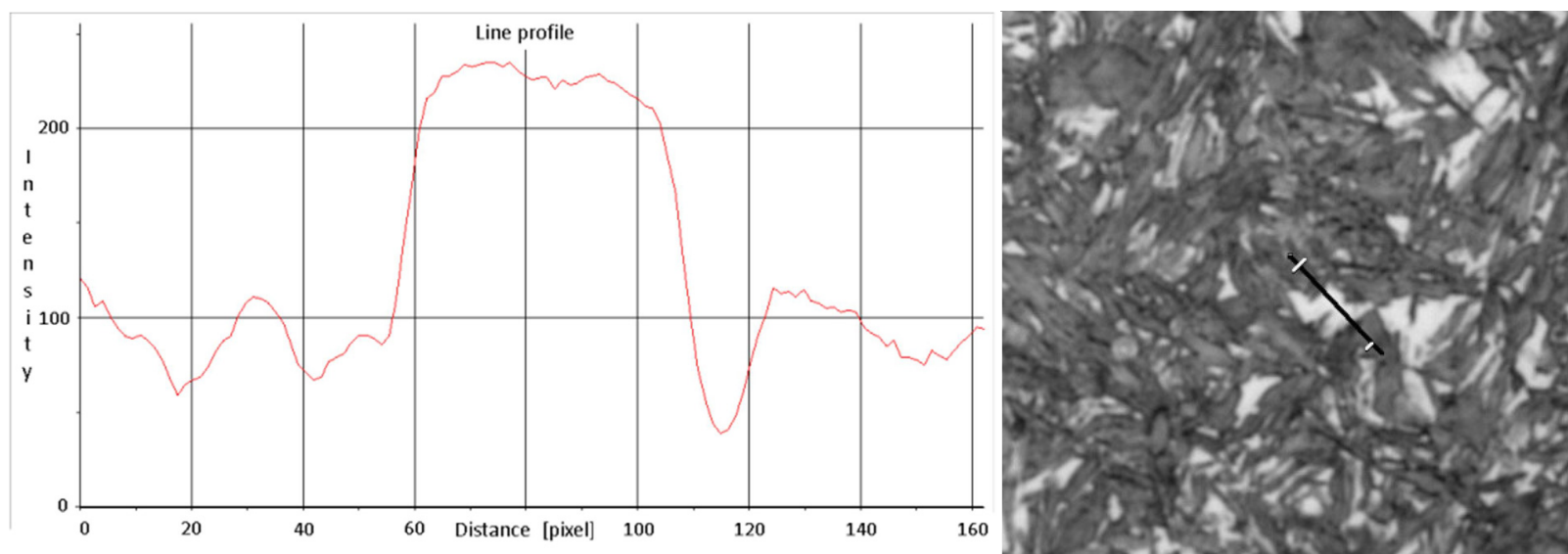

Fig. 2. Gray level profile and image structure of the sample without a delinearizing filter
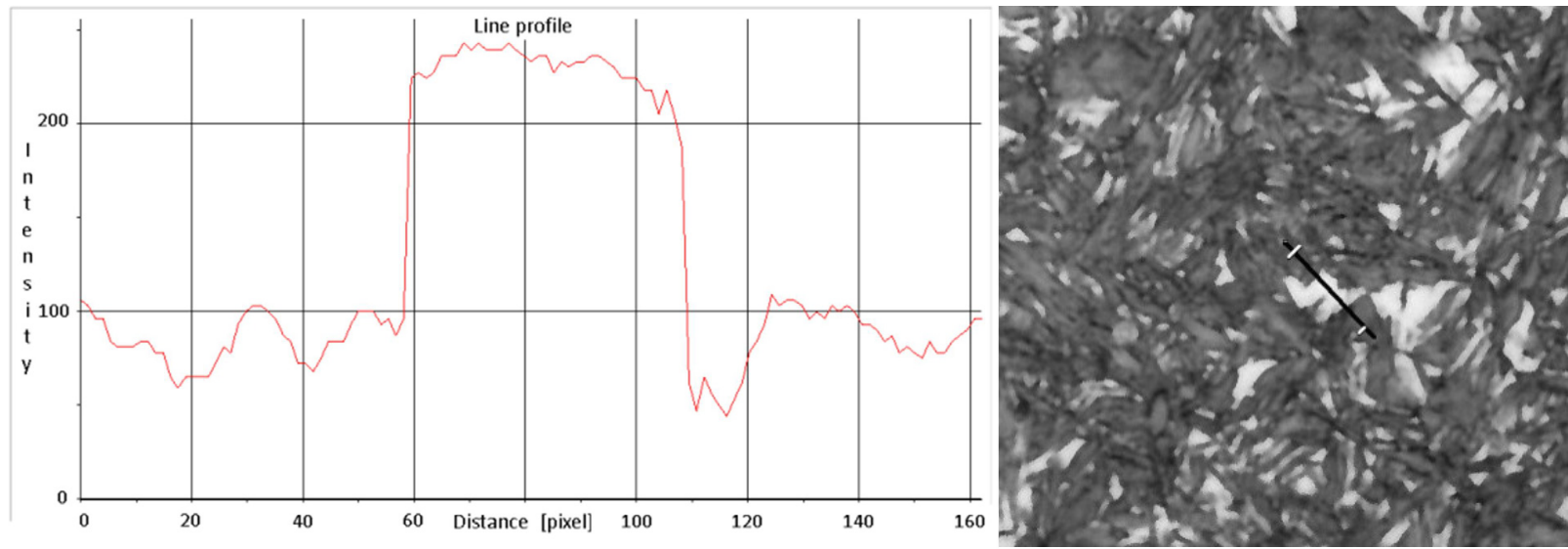

Fig. 3. Gray level profile and image structure of the sample after delinearizing filtering 


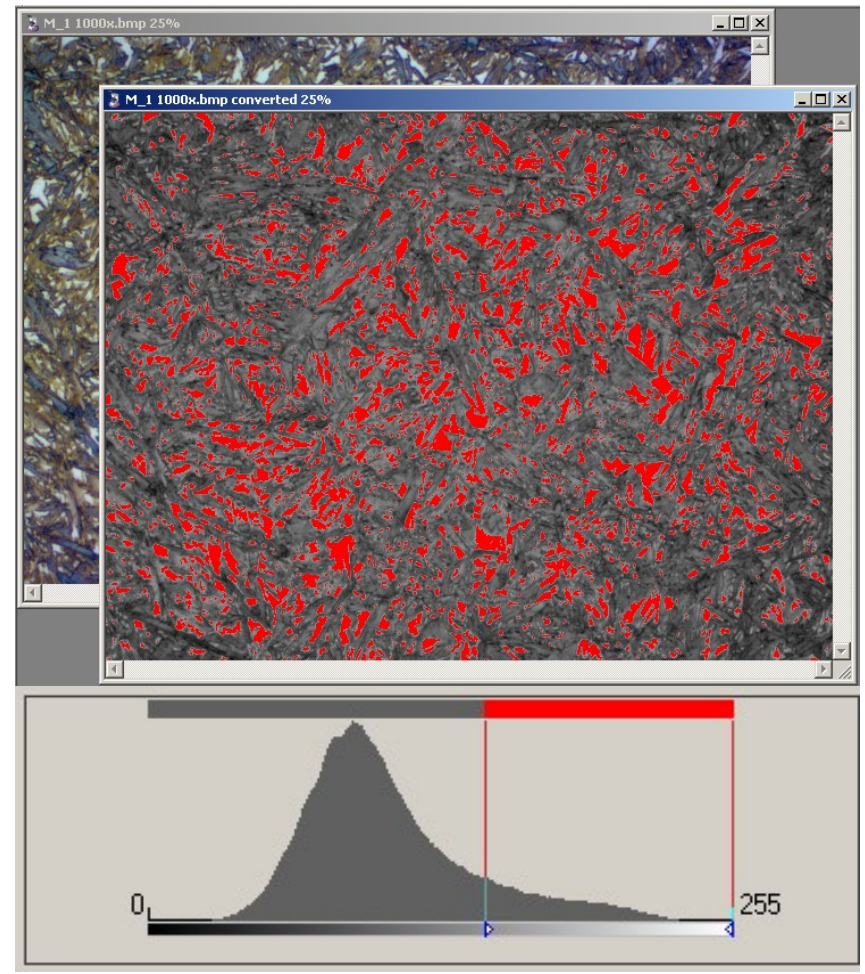

Fig. 4. Image editing, thresholding, mean gray scale level setting in the image without delinearization (convolution mask $5 \times 5$, threshold gray level 147)

the level 1 (default) to 0.5 was used. Images without using the delinearizing filter, with using the delinearizing filter and also with using the classical sharpening filter (convolution mask 5x5) were evaluated in the computer program. The threshold gray level in the delinearized image was determined as the mean value of two maxima on the histogram (Fig. 5 - the larger peak belongs to martensite and the minor peak belongs to retained austenite). In the case of evaluation without delinearization, threshold gray levels were determined subjectively whereas the histogram did not distinctly separate gray levels between martensite and retained austenite. The thresholding was performed manually for the best coverage of the retained austenite areas (Fig. 4). Similarly, thresholding was performed in the image edited using the classical sharpening filter.

\section{Results and discussion}

The determination of retained austenite content and the used techniques are showed in Table 1. The optimal gray level determined on the basis of the histogram is indicated in boldface. In order to detect the effect of the setting the threshold gray level the evaluation of retained austenite content was also carried out for values ten units below or above the optimal threshold gray level.
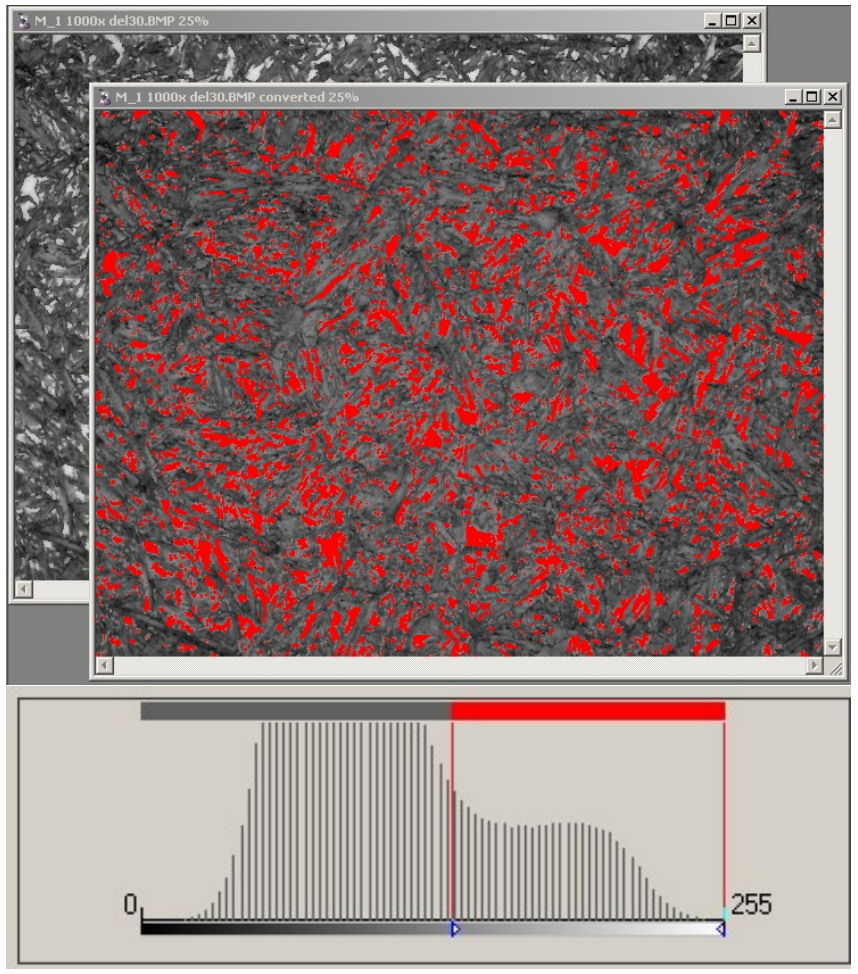

Fig. 5. Image editing, thresholding, mean gray scale level setting in the delinearized image (convolution mask $5 \times 5$, threshold gray level 136 ).

TABLE 1

Determination of the retained austenite content using different image editing methods (RA - retained austenite)

\begin{tabular}{|c|c|c|c|c|c|}
\hline \multicolumn{6}{|c|}{ Without image adjustment (without delinearising filter) } \\
\hline & $\begin{array}{l}\text { Threshold } \\
\text { gray level }\end{array}$ & $\begin{array}{c}\text { RA } \\
\text { Portion } \\
{[\%]}\end{array}$ & & $\begin{array}{l}\text { Threshold } \\
\text { gray level }\end{array}$ & $\begin{array}{c}\text { RA } \\
\text { Portion } \\
{[\%]}\end{array}$ \\
\hline \multirow{3}{*}{$\begin{array}{l}\text { gamma } 1.0 \\
\text { subjectively }\end{array}$} & & 17.6 & \multirow{3}{*}{$\begin{array}{l}\text { gamma } 0.5 \\
\text { subjectively }\end{array}$} & 0. & 18.1 \\
\hline & 147 & 13.9 & & 93 & 14.6 \\
\hline & 157 & 10.9 & & 103 & 11.7 \\
\hline \multicolumn{3}{|c|}{$\begin{array}{c}\text { Difference between min. and max.: } \\
6.7 \%\end{array}$} & \multicolumn{3}{|c|}{$\begin{array}{c}\text { Difference between min. and } \\
\text { max.: } 6.4 \%\end{array}$} \\
\hline
\end{tabular}

With delinearising filter, The threshold for he Sobel operator 30

\begin{tabular}{|c|c|c|c|c|c|}
\hline & $\begin{array}{l}\text { Threshold } \\
\text { gray level }\end{array}$ & $\begin{array}{c}\text { RA } \\
\text { Portion } \\
{[\%]}\end{array}$ & & $\begin{array}{l}\text { Threshold } \\
\text { gray level }\end{array}$ & $\begin{array}{c}\text { RA } \\
\text { Portion } \\
{[\%]}\end{array}$ \\
\hline \multirow{3}{*}{$\begin{array}{c}\text { matrix } 5 \times 5 \\
\text { gamma } 1.0 \\
\text { peaks } 84 \\
187\end{array}$} & 126 & 20.2 & \multirow{3}{*}{$\begin{array}{c}\text { matrix } 5 \times 5 \\
\text { gamma } 0.5 \\
\text { peaks } 29, \\
147\end{array}$} & 78 & 16.4 \\
\hline & 136 & 17.3 & & 88 & 14.3 \\
\hline & 146 & 15.0 & & 98 & 12.4 \\
\hline
\end{tabular}

Difference between min. and max.: $\quad$ Difference between min. and

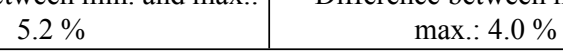

\begin{tabular}{|c|c|c|c|c|c|}
\hline \multicolumn{6}{|c|}{ Sharpening filter } \\
\hline & $\begin{array}{l}\text { Threshold } \\
\text { gray level }\end{array}$ & $\begin{array}{c}\text { RA } \\
\text { Portion } \\
{[\%]}\end{array}$ & & $\begin{array}{l}\text { Threshold } \\
\text { gray level }\end{array}$ & $\begin{array}{c}\text { RA } \\
\text { Portion } \\
{[\%]}\end{array}$ \\
\hline \multirow{3}{*}{$\begin{array}{l}\text { matrix } 5 \times 5 \\
\text { gamma } 1.0 \\
\text { subjectively }\end{array}$} & 126 & 19.6 & \multirow{3}{*}{$\begin{array}{c}\text { matrix } 5 \times 5 \\
\text { gamma } 0.5 \\
\text { subjectively }\end{array}$} & 88 & 14.0 \\
\hline & 136 & 15.8 & & 98 & 11.7 \\
\hline & 146 & 12.7 & & 108 & 9.8 \\
\hline \multicolumn{3}{|c|}{$\begin{array}{l}\text { Difference between min. and max.: } \\
6.9 \%\end{array}$} & \multicolumn{3}{|c|}{$\begin{array}{l}\text { Difference between min. and } \\
\max .: 4.2 \%\end{array}$} \\
\hline
\end{tabular}


It is evident from Table 1 that the setting of the correct threshold gray level has a significant effect on the resulting values of retained austenite content. Each image editing, whether the use of filters or gamma function, has got an impact on the final value of the retained austenite content. The universal setting of the gamma function to the value of 0.5 is not always ideal, because in the case of using delinearizing and sharpening filter, the lower value of the retained austenite content was found. When setting the gamma function to 0.5 , the difference between the minimum and maximum value of the retained austenite content for different threshold gray levels was smaller than for the gamma function equal to 1.0. The use of the delinearizing filter appears to be preferred. The lowest difference between the minimum and maximum values of the retained austenite content was found in this case, depending on the threshold gray level. However, there is not a universal technique of picture editing and threshold gray level setting, because each image is unique and influenced by sample preparation, microscope setting, image editing techniques and evaluation methods.

This work remained to only evaluate the proportion of retained austenite by image analysis. Using other methods for evaluation of retained austenite was disclosed by [5-9]. The higher value of retained austenite was determined by X-ray diffraction analysis (for this specimen $25.7 \%$ RA). Observed differences in the proportion of retained austenite correspond to trends that are described in the literature [4,7-9].

\section{Conclusion}

An important role in the use of image analysis plays the sample preparation, especially the intensity of etching the sample. From the results of the retained austenite content, assessment can be concluded that this is a very sensitive method and that there is no universal technique of image editing and setting the gray levels to evaluate the differently prepared samples. Each image is unique and influenced by sample preparation, setting the microscope, image editing techniques, and evaluation.

\section{Acknowledgement}

This paper was created with the contribution of the projects Student Grant Competition SP 2016/103 Specific research in metallurgy, materials and process engineering, and SP2016/111 Study of relationships between structural parameters and properties of advanced materials. We are also thankful for the contribution of the Project No. LO1203 "Regional Materials Science and Technology Centre - Feasibility Program" funded by Ministry of Education, Youth and Sports of the Czech Republic.

\section{REFERENCES}

[1] G. Krauss, Steels: heat treatment and processing principles, 1990 ASM International.

[2] K.E. Thelning, Steel and his treatment, 1984 Butterworths, London.

[3] L. Wojnar, Image analysis Application in Materials Engineering, 1999 CRC Press, New York.

[4] H. Ma. The Quantitative Assessment of Retained Austenite in Induction Hardened Ductile Iron [online]. Electronic Theses and Dissertations, University of Windsor, 2012. http://scholar. uwindsor.ca/cgi/viewcontent.cgi?article=1196\&context=etd

[5] S.H. Magner, R J. De Angelis, W.N. Weins, J. D. Makinson, JCPDS-International Centre for Diffraction Data 2002, Advances in X-ray Analysis. 45, 92-97 (2002).

[6] Y.Y. Su, L.H. Chiu, T.L. Chuang, C.L. Huang, C.Y. Wu, K.C. Liao, Adv. Mat. Res. 482, 1165-1168 (2012).

[7] F.L. Sicupira, M.J.R. Sandim, H.R.Z. Sandim, D B. Santos, R.A. Renzetti, Mater. Charact. 115, 90-96 (2016).

[8] L. Ryde, Mater. Sci. Tech. 22, 1297-1306 (2006).

[9] P. Váňová, J. Sojka, A. Volodarskaja, K. Jokešová, P. Purtátor, A. Spinu, in: Metal 2016, Tanger Ltd. Ostrava 2016. 